\title{
Artificial Intelligence based 6G Intelligent IOT: Unfolding an Analytical Concept for Future Hybrid Communication Systems
}

\author{
Muhammad Haroon Siddiqui ${ }^{1+}$, Kiran Khurshid ${ }^{1}$, Imran Rashid ${ }^{1}$ and Adnan Ahmed Khan ${ }^{2}$ \\ ${ }^{1}$ Electrical Engineering Department, National University of Sciences and Technology, Islamabad, Pakistan. \\ ${ }^{2}$ Computer Software Engineering Department, National University of Sciences and Technology, Islamabad, \\ Pakistan.
}

\begin{abstract}
The Internet of Things (IOT) is a new paradigm for wireless communication networks with access to artificial intelligence (AI). The conventional cellular network is experienced with two major issues such as spectrum scarcity and insufficient intelligent autonomous competency. These problems are required to be addressed otherwise it will obstruct the massive deployment of next generation applications. The concept of the next generation communication network, creates the opportunity to design a network with number of sensors in the paradigm of IOT to process large data. In this paper, we present a novel $6 \mathrm{G}$ intelligent IOT paradigm to optimize communication channels and process big data intelligently. Firstly, we present the proposed architecture of $6 \mathrm{G}$ intelligent IOT paradigm, then we discuss the enabling technologies in relation to our proposed concept. In $6 \mathrm{G}$ scenario, $\mathrm{AI}$ is not very effective as compared to the physical layer (PHY), however, it provides flexibility and agility to the air interface with enhanced efficiency. We discuss AI based 6G air interface architecture, based on our proposed paradigm. Finally, we evaluate the results through simulation by comparing our propose paradigm with 5G-IOT and 5G intelligent IOT. Simulation results confirm that our proposed paradigm out performs the others.
\end{abstract}

Keywords: Artificial intelligence, cognition cycle, deep learning, super massive-MIMO, 6G.

\section{Introduction}

5G wireless network is considered, a key enabler, for intelligent information society of 2020. Extensive efforts are being made by 3rd generation partnership project (3GPP) to encourage further development of $5 \mathrm{G}$ technologies. In the meantime, IEEE 8011 ax standard for wireless local area network is being introduced [1]. It is anticipated that even after introduction of millimeter wave (mm-wave) and massive multi input multi output (MIMO) (with large scale arrays of antennas), $5 \mathrm{G}$ can maximum achieve $20 \mathrm{~Gb} / \mathrm{s}$ transmission for end user. The latest applications such as internet of things (IoT), artificial intelligence (AI), cognition cycle (CC), IP multimedia subsystem (IMS), vehicular network and machine learning, will depict the next generation of cellular network, viz: 6G. Investigations and research on 6G wireless networks has also been made a part of agenda to meet the needs for the intelligent information society 2030 [2], [3]. China has also introduced a plan "broadband communication and new networks" for 2030 and beyond. The university of Oulu (Finland), has launched 6G research program viz: 6G-enabled wireless smart society and ecosystem (6Genesis) [4], [5], which will explore the avenues for the development of $6 \mathrm{G}$ standards. Various projects related to beyond-5G (B5G), have been sponsored by european commission's horizon 2020 program. In United States, federal communication commission (FCC) has already started research on $6 \mathrm{G}$ and launched $\mathrm{THz}$ band. Intelligent information society 2030 is envisaged highly digitized, intelligent, autonomous, globally data driven and unlimited wireless connectivity [6]. Fig.1 shows the resource utilization of $6 \mathrm{G}$ based on time-frequency-

\footnotetext{
${ }^{+}$Muhammad Haroon Siddiqui. Tel.: + 923335125126.

E-mail address: haroon@mcs.edu.pk
} 
space. The concept of $6 \mathrm{G}$ will be the key enabler in order to achieve this blueprint. It is anticipated that $6 \mathrm{G}$ will be autonomous with intelligence and consciousness like human. Moreover, $6 \mathrm{G}$ technology will:

- Support ultra-high definition videos with extremely high throughput

- Allow highly low latency communications such as $10 \mu$ s, especially for industrial internet [7]

- Support Nano technologies which include internet of nano-things, implantable nano-sensors and nano-devices with extremely high energy efficiency

- Support space and deep sea communications

- Enhance and support existing 5G key applications.

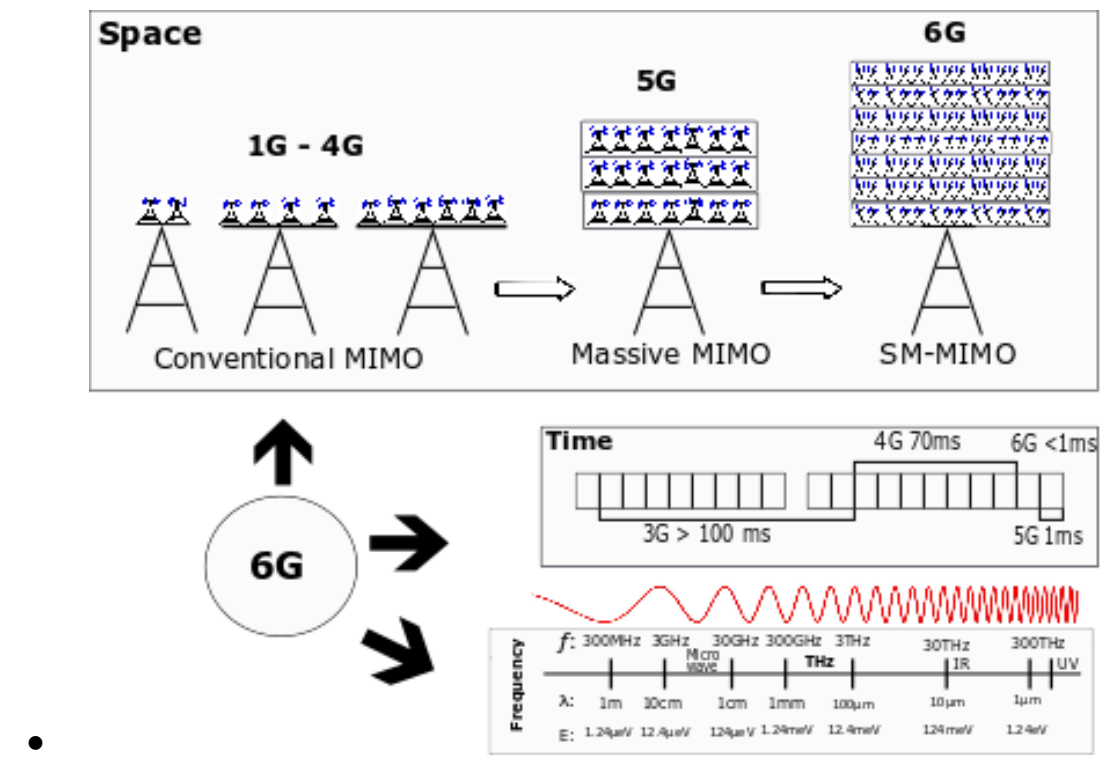

Fig. 1: An elucidation of resource utilization of $6 \mathrm{G}$ based on time-frequency space.

The ever growing attraction especially in the domain of $6 \mathrm{G}$, IoT, AI and big data, draws the attention of researchers to fully comprehend their conceptual structure, future potential and challenges. Likewise, the future concept of communication systems with enormous sensors, demands convergence of key technologies to produce novel paradigm. 6G intelligent IoT paradigm, is the convergence of intelligence, internet and things. The concept of $6 \mathrm{G}$ is a backbone for realization of future IoT technology as IoT applications require to handle a large number of heterogeneous devices. A network of such ever-increasing number of devices can benefit from 6G technology. 6G technology can provide the connectivity, desired network standards and architecture for real time processing of the massive data with low latency rate. In this paper, we make an endeavour to propose an architecture of $6 \mathrm{G}$ intelligent IoT and evaluate its major components. Then we introduce some of the key and enabling technologies for $6 \mathrm{G}$ intelligent IoT with their application on proposed paradigm. Finally, we evaluate the performance of proposed paradigm on the basis of some performance metrics.

\section{The Architecture of $6 \mathrm{G}$ Intelligent IoT}

Fig. 2 shows the architecture of $6 \mathrm{G}$ intelligent IoT, which is based on existing cellular network. It is a general cellular network with the latest emerging technologies, such as, super massive (SM)-MIMO, ultradense static and mobile cells networks [8]. It also presents the BSs and a cloud in 6G intelligent IoT paradigm. The concept of device-to-device (D2D) communication [9] and small cell access points are also integrated in this architecture. $6 \mathrm{G}$ intelligent IoT paradigm comprises of three major components.

\subsection{Intelligent Ultra High Speed Processing Core (IUSPC)}

6G intelligent IoT paradigm is envisaged for autonomous, intelligent and without human intervention applications. To make this component more intelligent and avoid repetition to reduce latency time, cognition cycle (CC) is used for learning and previous memory. AI techniques, such as swarm intelligence, genetic algorithm, reinforcement learning, and multi-layer perceptron are being used to implement CC [10]. The 
other two components in the cloud include intelligent computing module (ICM) and execution module (EM). ICM comprises of several intelligent computing system and known as the nerve center. AI technique (deep learning) is used for the online learning realization. In all ICM, multiple servers are grouped together for intelligently processing of massive data. This arrangement allows ICM to operate independently and efficiently. ICM is also responsible to process the data intelligently from the Intelligent Base station (IBS) and passing the instructions to the IBS accordingly. CC plays a vital role in the scheme in order to reduce the latency rate as learning from the past experiences, save the processing time required for computation.

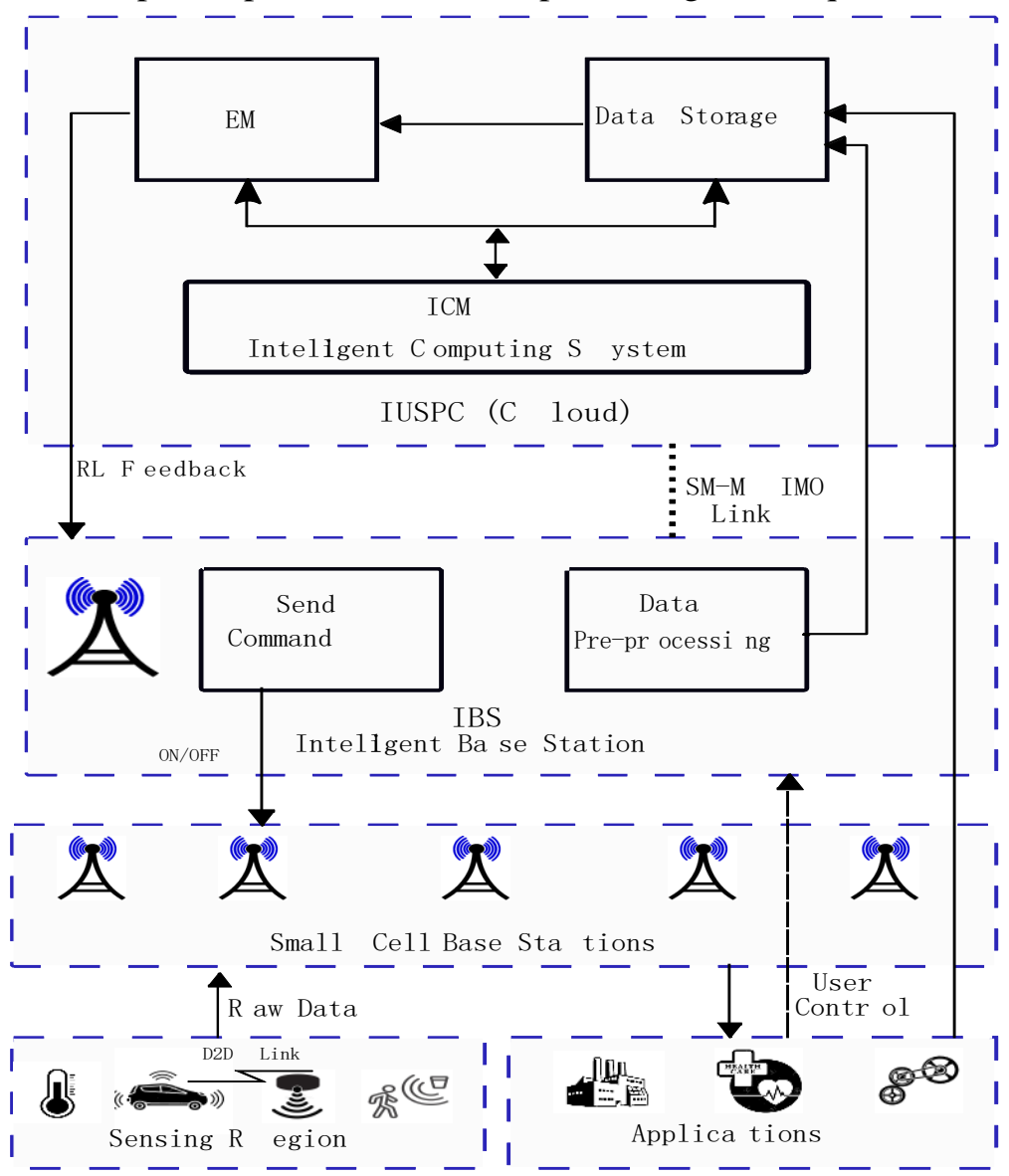

Fig. 2: 6G intelligent IoT architecture.

\subsection{IBSs}

An IBS is consisted with all requisite requirements to connect IUSPC with the sensing regions. Raw data is processed at IBS, then processed data is transmitted to IUSPC. On the basis of feedback received from IUSPC, more intelligent schemes are implemented. It is anticipated that in future communications especially in heterogeneous operating environment, massive unprocessed data, transmitted to the BS through ultradense small cell networks, is required to be processed at high cost. Hence, unprocessed data must be preprocessed redundantly. The IBS receives the instructions from the IUSPC for intelligently execution, however, if the channel is disconnected the sensing region will stop transmission. CC, is embedded in IBS and helps in selecting (through learning from past experience) the correct unoccupied channel at right time to prevent unnecessary transmission. This will reduce the traffic load, enhance the data processing speed and improve the energy efficiency.

\subsection{Sensing Region}

This region has sensors in large numbers, which are used to compute or determine the physical quantity and convert it into digital signal for processing. The $6 \mathrm{G}$ terahertz $(\mathrm{THz})$ will support more effectively the emerging applications such as D2D communication [11] and internet of vehicle (IoV) [12]. These applications emerge in the physical world or sensing region. The main function of the sensing region is to observe the state of particular domain. On the basis of the nature of assignment, these regions are divided into mobile sensors region (deals with mobile devices) and fixed or static sensors region. These regions assist 
IoT devices to interact with the environment and collect the sensing information. This information is subsequently transmitted to $\mathrm{THz}$ small cell networks and then to IBS for further processing in $6 \mathrm{G}$ environment.

\section{Promising Technologies to Implement 6G Architecture}

\subsection{Message Queuing Telemetry Transport (MQTT) Protocol}

In ubiquitous communication environment, IoT devices communicate through MQTT protocol [13]. The MQTT uses TCP/IP for network connections and can send simultaneous messages to multiple receivers. In an intelligent IoT based 6G architecture, MQTT protocol is used for interconnection of the various components [14]. MQTT protocol has some inherent qualities due to which the chances of loss and duplication of the data information is reduced, hence, results in efficient use of the radio resources.

\section{2. $\mathrm{THz}$}

$\mathrm{THz}$ communications [15] band (in the range of 0.1-10 THz) uses richer spectrum resources than that of mm-wave band in 5G. It can take the benefits of both light and electromagnetic waves. In $6 \mathrm{G}$ architecture, $\mathrm{THz}$ communication is expected to provide multi-Tb/s data transmission to various emerging applications at sensing region [16]. Moreover, the benefits, anticipated, to achieve through $\mathrm{THz}$ communication are:

- It can support massive bandwidth demand as envisage in 6G technology and provide multi-Tb/s transmission.

- Due to shorter wave length, THz can integrate multiple antennas, hence can provide thousands of beams. It is anticipated that 10000 antennas can be accommodated in THz based IBSs which results in achieving extreme narrow beams and ultra-high data transmission.

- Inter-cell interference free and secure communication due to highly directional transmission.

\subsection{SM-MIMO, Large Intelligent Surface and Holographic Beamforming}

MIMO, with more than ten thousand of antennas, enhances the capacity manifold and provides reliable transmission by diversity [17]. It also reduces the propagation losses by narrow beamforming. In a $6 \mathrm{G}$ intelligent IoT paradigm, SM-MIMO is expected to offer following benefits:

- It improves energy efficiency and enhances the latency rate.

- Increases network throughput.

- Massive access communication can be achieved by combining SM-MIMO and non-orthogonal multiple access (NOMA) schemes.

- Ultra-narrow beams reduce propagation losses as well as lessen the co-channel inter-cell interference.

\subsection{Big Data Mining}

In $6 \mathrm{G}$ Intelligent IoT paradigm, real time data processing is required, hence, big data generated in this scenario is altogether different from conventional data. Data collection signals from small cells is broadcasted to the network management. Data is collected from both mobile and fixed sensor regions according to the instructions. Then raw data is sent to the IBSs where data is pre-processed. The methods used for pre-processing, include integration, cleansing and redundancy. Data integration gathers data from all the sources and combines it. In cleansing, irrelevant, incomplete and unwanted data is deleted. Finally, in redundancy, redundant data is removed and compression is used for efficient utilization of spectral resources.

\subsection{Deep Learning}

Deep learning is a branch of machine learning, which is based on learning of data representations from different architectures. Deep learning has already been applied to many applications, which has proved to be an efficient technique. It is regarded as a key step toward actual AI. Massive data is produced with the development of intelligent IoT in a $6 \mathrm{G}$ paradigm. Hence, big data mining and deep learning can render very effective and efficient services in this scenario. Fig.2 also shows that ICM and EM is placed at the center, so that data can be processed automatically with intelligent algorithms. 
Various independent intelligent computing systems are designed to process unique data intelligently, such as a vehicle number plate detection system, a human face identification system, and any specific oddity detection system. They implement deep learning algorithms and act within specific scenario as an ICM. Every ICM is a unique system which processes distinct data, where the processed data information is readily available to be shared among systems. ICMs collect real time data from various sources, process it, and forward it to EM for further disposal before storing it locally or being sent it to the users or in the cloud.

\subsection{Reinforcement Learning}

Reinforcement learning presents the scenario of the states in a particular environment by taking various actions and receiving rewards. One of the key feature of reinforcement learning is to find the optimal solution by investigating an unknown environment. The major three components in this perspective involved in the procedure are: agents, rewards and policies. In a $6 \mathrm{G}$ intelligent IoT paradigm, an agent is described as the collection of IBSs and IUSPC. Data from all the sensing regions are fed as an input, whereas, channel's states activity from the EM, is controlled by the command which is the output of an agent. In a big data environment, reward is generated for each command. To ensure the quality of experience (QoE) of users, CC keeps the track of channel occupancy.

A policy is formulated, with corresponding control command for implementation, at IUSPC. The essence of each policy, is to provide an efficient and accurate judgment. After successful evaluation process, policy is introduced to the communication system. In an EM, multiple probability coefficients are defined according to the various applications scenarios. The probability coefficients specify the significance of each ICM. After initialization of probability coefficients and processing of the data at ICM, processed data, is multiplied by their corresponding coefficients. Then the result is compared with predesignated threshold in EM. If the resulting quantity exceeds the threshold, then a control command is sent to IBS for closing the transmission and restricting other unwanted data to upload.

Reinforce learning technique, assists EM in automatic learning of the probability coefficients and thresholds for various application as well as intelligently online execution. Likewise, ICM can also learn automatically in various applications environment and can process the data online, intelligently and accurately. It has been experienced that through online techniques, automatic and intelligent performance can be achieved, which is definitely far better than the manual adjustment of threshold.

\section{7. $\mathrm{CC}$}

$\mathrm{CC}$ is the concept of learning from the previous actions for optimal outcomes with the passage of time. There are basic five steps are involved in implementing CC, Fig.3 shows various stages of CC [18], [19]. In first stage data is collected from sensor regions and is observed thoroughly. After observation stage, received data is analyzed to identify the pattern. In third stage, course of action is adopted from various options. In fourth step, actions are executed. Lastly, it learns the knowledge from the outcome of executed actions for future compliance.

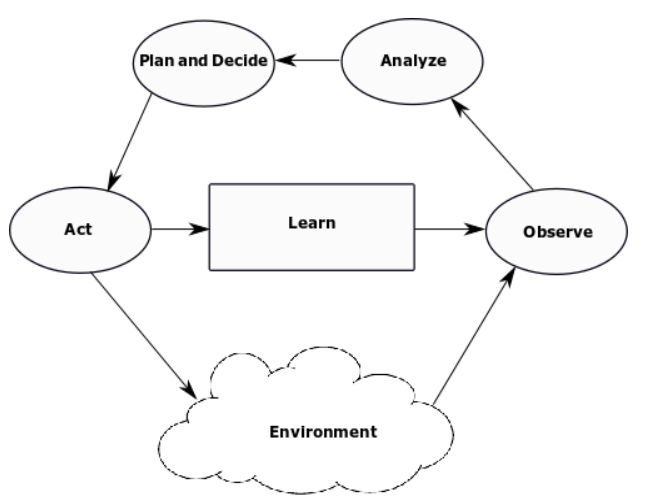

Fig. 3: An illustration of cognition cycle.

\section{AI Based 6G Interface}

Table-I explains the air interface implementation for $6 \mathrm{G}$ intelligent IoT paradigm. AI will notably revolutionize the architect of $6 \mathrm{G}$ air interface. 
Table 1: Ai Impacts on $6 \mathrm{~g}$ Network Functions

\begin{tabular}{|c|c|c|c|}
\hline & Functions & AI Algorithms & Descriptions \\
\hline 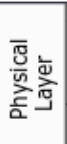 & $\begin{array}{l}\text { - Reliable Data Transmission } \\
\text { Channel coding, modulation, } \\
\text { MIMO precoding and OFDM } \\
\text { - Channel Estimation }\end{array}$ & $\begin{array}{l}\text { - DNNs } \\
\text { - Autoencoder } \\
\text { CNNs } \\
\text { - CCNNs } \\
\text { K-Means }\end{array}$ & $\begin{array}{l}\text { - Revamp end-to-end PHY } \\
\text { architecher reduces complexity } \\
\text { level of MIMO OFDM receiver } \\
\text { - During high mobility enhances } \\
\text { PHY performance }\end{array}$ \\
\hline 恙㐫 & $\begin{array}{l}\text { Frame flowing operations } \\
\text { flow control synchronization, } \\
\text { data packets queing, scheduling } \\
\text { power control, error correction } \\
\text { and flow control etc }\end{array}$ & $\begin{array}{l}\text { DNNs } \\
\text { Supervised learning } \\
\text { - Deep learning } \\
\text { Reinforcement } \\
\text { Learning } \\
\text { - Transfer Learning } \\
\text { - Q- Learning }\end{array}$ & $\begin{array}{l}\text { Optimal user scheduling } \\
\text { Improve network performance } \\
\text { Channel Estimation } \\
\text { Traffice Prediction } \\
\text { Enhances radio resource } \\
\text { efficiency } \\
\text { Optimal retransmission } \\
\text { redundancy } \\
\text { Reduction in retransmission } \\
\text { overhead }\end{array}$ \\
\hline 美离 & $\begin{array}{l}\text { - Responsible for management } \\
\text { of: } \\
\text { Riuting,Mobility,RRC connection } \\
\text { and load } \\
\text { - BS association } \\
\text { - BS clustring }\end{array}$ & $\begin{array}{l}\text { DNNs } \\
\text { K-Means } \\
\text { - Unsupervised } \\
\text { Learning } \\
\text { - Supervised learning } \\
\text { - Reinforcement } \\
\text { Learning } \\
\text { - Q- Learning }\end{array}$ & $\begin{array}{l}\text { Optimizes serving cells } \\
\text { - Optimizes multiple connectivity } \\
\text { - Mobility prediction } \\
\text { - Optimizes handover } \\
\text { - Optimizes data transmission } \\
\text { paths } \\
\text { - Optimizes BS clustring } \\
\text { - Controlling size of cluster } \\
\text { in dynamic network scenerios }\end{array}$ \\
\hline
\end{tabular}

\subsection{Physical-Layer (PHY)}

It is the most significant layer in achieving the reliable and rapid data transmission over wireless channels. At transmitter side, various modules perform operations such as coding, modulation, orthogonal frequency division multiplexing (OFDM) and MIMO precoding. Likewise, reverse operations are performed at receiver side to recover the original data. In 6G intelligent IoT paradigm, an AI based end-to-end architecture is designed, in which auto-encoders from deep learning can be used to perform processing of the data intelligently. However, to avoid complexity and make this scheme more practical, AI application is designed independently which enhances the functions of PHY. In deep learning method, convolution neural networks (CNNs) can perform signal classification and channel decoding, whereas OFDM is built with complex CNNs (CCNNs). Signal detection and channel estimation can be performed through deep neural networks (DNNs).

\subsection{Data-Link Layer}

AI can enhance the sub-layers of data-link layers including medium-access control, packet dataconvergence protocol, radio-link control and service data adaptation protocol. AI enabled resource allocation, is capable of selecting the most appropriate scheduling for users with (or without) traffic prediction [20]. Security enhancements can be achieved by adopting suitable AI security algorithms [21]. In intelligent IoT 6G scenario AI security algorithms are very effective due to the short packets. Another key feature of AI, is to optimize the operation of automatic repeat request (ARQ) and hybrid ARQ. It can lessen the retransmission overheads and improve the reliability of data transmission.

\subsection{Network Layer}

Network layer generally renders IBS and user specific functions which include traffic load balancing, radio resource control (RRC) and mobility management. AI techniques enable user to choose optimal serving cells, multiple connectivity and various other features, which can guarantee the continuity of service. Likewise, with AI, IBSs can balance the traffic load and improve the network robustness.

\section{Performance Evaluation and Analysis}

The basic aim of an $6 \mathrm{G}$ intelligent IoT paradigm is to achieve intelligently big data processing and enhance the efficiency of communication channels to optimum. There are quite number of performance evaluation metrics, to evaluate the actual performance of a $6 \mathrm{G}$ communication networks, such as complexity implementation, spectral efficiency, energy efficiency, latency, data rate, QoS, processing speed and many more. It is very hard to incorporate all the performance metrics to evaluate the efficacy of $6 \mathrm{G}$ intelligent IoT due to high complexity level. However, we chose effective utilization of channels (EUOC) as a performance indicator to assess our simulation results. The EUOC is computed by $\mathrm{k} / \mathrm{m}$, where $\mathrm{k}$ is the group of various service data and $\mathrm{m}$ is the group of valid service data. Simulations of 5G IoT, $5 \mathrm{G}$ intelligent IoT and $6 \mathrm{G}$ 
intelligent IoT are shown in figures with blue, red and black line respectively. In Fig.4, EUOC performances of 5G IoT and 5G intelligent IoT, are compared. Result shows that 5G intelligent IoT performs better than 5G IoT. Likewise, Fig.5 exhibits the performances of 5G IoT and 6G intelligent IoT paradigm. It is very evident from the simulation results that proposed $6 \mathrm{G}$ intelligent IoT outperforms the $5 \mathrm{G}$ IoT in terms of EUOC performance. In Fig.6, the EUOC performances of 5G intelligent IoT and $6 \mathrm{G}$ intelligent IoT are compared. Simulation results confirm that the proposed architecture shows significantly better results as compared to 5G intelligent IoT. In Fig.7, EUOC performances are compared for three scenarios, including $5 \mathrm{G}$ IoT, $5 \mathrm{G}$ intelligent IOT and $6 \mathrm{G}$ intelligent IoT. Experimental results show that performance of $6 \mathrm{G}$ intelligent IoT is the most effective.

Effective Utilization of Channels Curve in Random Environment

Effective Utilization of Channels Curve in Random Environment

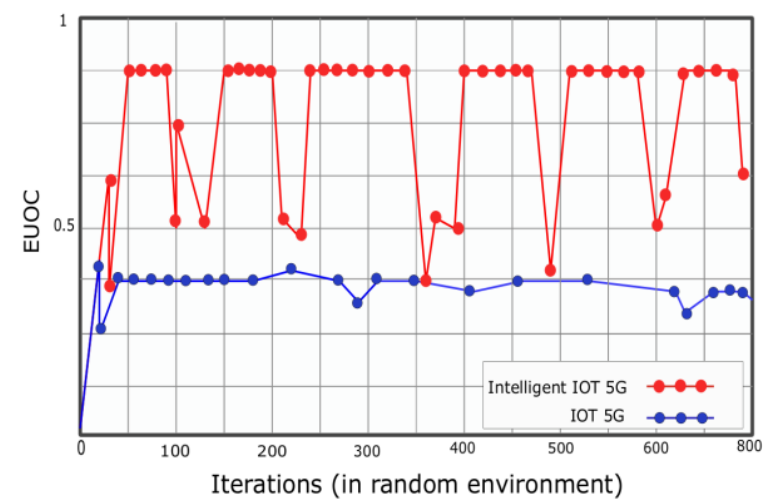

Fig. 4: Performance evaluation curves of effective utilization of channels (EUOC).

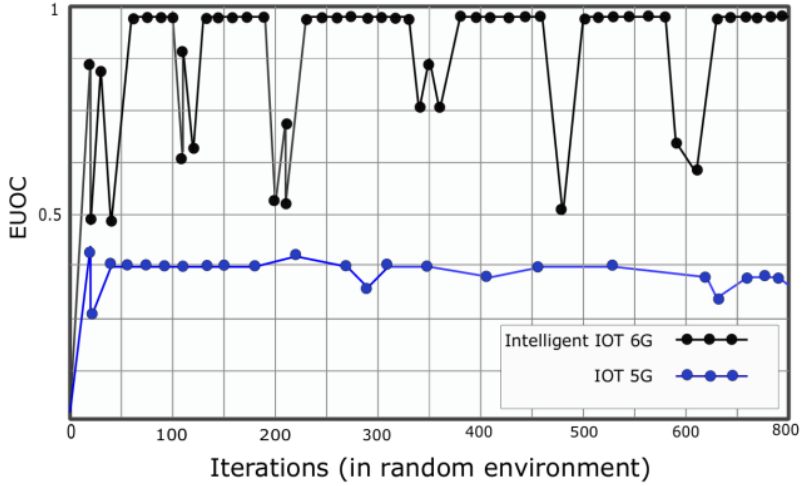

Fig. 5: Performance evaluation curves of effective utilization of channels (EUOC).

Effective Utilization of Channels Curve in Random Environment

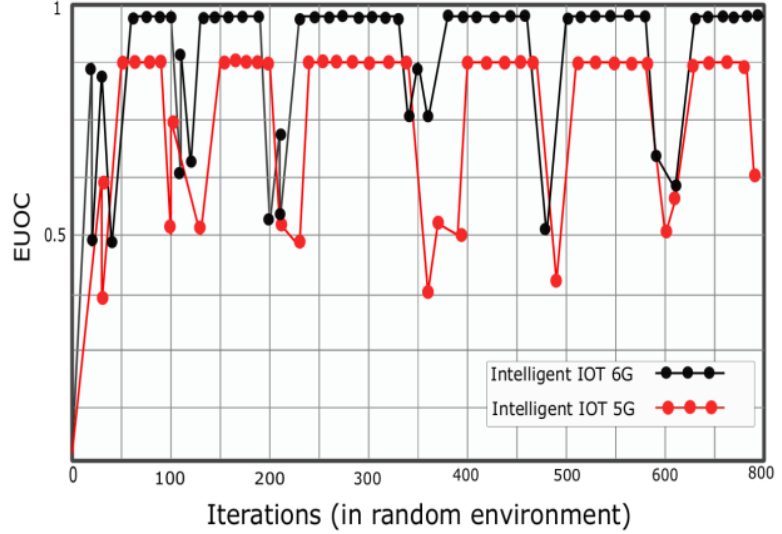

Fig. 6: Performance evaluation curves of effective utilization of channels (EUOC).

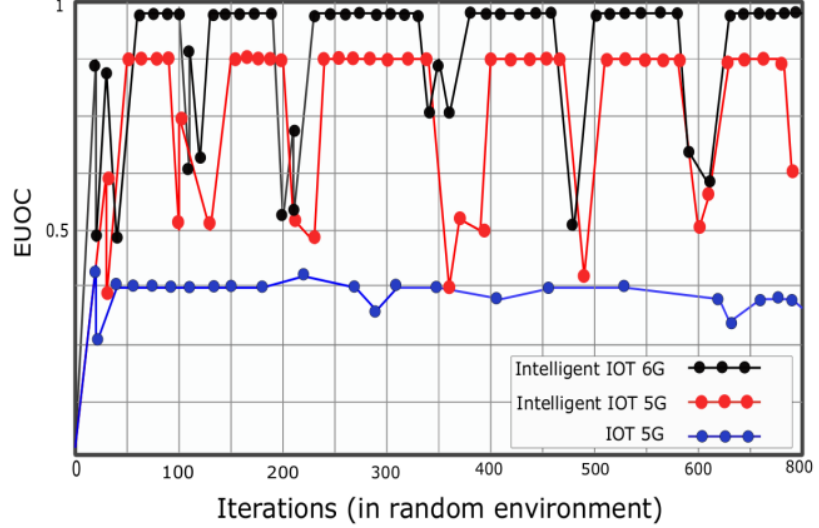

Fig. 7: Performance evaluation curves of effective utilization of channels (EUOC).

\section{Conclusion}

We have presented a novel concept of the $6 \mathrm{G}$ intelligent IoT, which converges $6 \mathrm{G}$, IoT and emerging technologies to an innovative paradigm. Our proposed architecture, can cater to the ever increasing demand of future communication network, especially in the domain of optimization of communication channels and processing of massive data. First, we have presented the three main elements of $6 \mathrm{G}$ intelligent IoT paradigm viz: IUSPC, IBS and sensing regions. Then we have introduced some enabling technologies with application to $6 \mathrm{G}$ intelligent IoT, such as MQTT protocol, $\mathrm{THz}$ band, $\mathrm{CC}$, big data mining, deep learning and reinforcement learning. AI based air interface has also been presented with an application to the $6 \mathrm{G}$ intelligent IoT framework. Finally, we have evaluated the experimental results of proposed architecture on the basis of performance indicators. We hope that this work gives an innovative concept on IoT, in which emerging technologies are applied to the collaborative paradigm of $6 \mathrm{G}$ and IoT. 


\section{References}

[1] E. Khorov, A. Kiryanov, A. Lyakhov, and G. Bianchi, "A tutorial on IEEE 802.11ax high efficiency WLANs", IEEE Commun. Surv. Tut. ,vol. 21, no. 1, pp. 197-216, 1st Quarter 2019.

[2] K. David and H. Berndt, “6G vision and requirements: Is there any need for beyond 5G?", IEEE Veh. Technol. Mag., vol. 13, no. 3, pp. 72-80, Sept. 2018.

[3] R. Li, "Network 2030: Market drivers and prospects" In Proc. 1st International Telecommunication Union Workshop on Network 2030, Oct. 2018. [Online]. Available: https://www.itu.int/en/ITU-T/Workshops-andSeminars/201810/Documents/Richard_Li_Presentation.pdf.

[4] A. Pouttu, "6Genesis-Taking the first steps towards 6G", In Proc. IEEE Conf. Standards Communications and Networking, 2018. [Online]. Available: csen2018.ieee-cscn.org/files/2018/11/AriPouttu.pdf.

[5] Z. Baiqing, Z. Xiaohong, W. Jianli, L. Xiaotong, and Z. Senlin, 'Photonics defined radio-A new paradigm for future mobile communication of B5G/6G", In Proc.6th Int. Conf. Photonics, Optics and Laser Technology, pp. 155-159, 2018.

[6] M. Latva-aho, "Radio access networking challenges towards 2030", 1st International Telecommunication Union Workshop on Network 2030, Oct. 2018. [Online]. Available: https://www.itu.int/ en/ITU-T/Workshops-andSeminars/201810/Documents/Matt_Latvaaho_Presentation.pdf.

[7] "Unified Architecture for Machine Learning in 5G and Future Networks", International Telecommunication Union-Telecommunication Standardization Sector, Technical Specification ITU-T FG-ML5G-ARC5G, Jan. 2019.

[8] S. H. Shah and I. Yaqoob, “A Survey: Internet of Things (IOT) Technologies, Applications and Challenges", Smart Energy Grid Engineering, pp. 381-85, Aug. 2016.

[9] L. D. Xu, W. He, and S. Li, “Internet of Things in Industries: A Survey", IEEE Trans. Industrial Informatics, vol. 10, pp. 2233-43, Jan. 2014.

[10] J.Qadir, “Artificial intelligence based cognitive routing for cognitive radio networks", Artif. Intell. Rev., vol. 45, no. 1, pp. 25-96, Jan. 2016.

[11] X. Liu, Z. Li, N. Zhao, W. Meng, G. Gui, Y. Chen and F. Adachi, "Transceiver Design and Multihop D2D for UAV IoT Coverage in Disasters", Internet of Things Journal IEEE, vol. 6, no. 2, pp. 1803-1815, 2019.

[12] Fatima Haouari, Ranim Faraj, Jihad M. AlJa'am, "Fog Computing Potentials Applications and Challenges", Computer and Applications (ICCA), pp. 399-406, 2018.

[13] A. Niruntasukrat et al., "Authorization Mechanism for MQTT-based Internet of Things", IEEE ICC Wksps., pp. 290-95, July 2016.

[14] A. Schmitt, F.Carlier and V.Renault, "Dynamic bridge generation for IoT data exchange via the MQTT protocol", Procedia Computer Science, vol. 130, pp. 90-97, 2018.

[15] JA. -A. A. Boulogeorgos et al., "Terahertz technologies to deliver optical network quality of experience in wireless systems beyond 5G", IEEE Commun. Mag., vol. 56, no. 6, pp. 144-151, June 2018.

[16] Zan Li, Lei Guan, Chenxi Li and Ayman Radwan, "A Secure Intelligent Spectrum Control Strategy for Future THz Mobile Heterogeneous Networks", IEEE Communications Magazine, vol.56, no.6 pp. 116 - 123, 2018.

[17] E. Björnson, L. Sanguinetti, H. Wymeersch, J. Hoydis, T. L. Marzetta, "'Massive MIMO is a reality-What is next? Five promising research directions for antenna arrays", 2019. [Online]. Available: https://arxiv .org/abs/1902.07678

[18] C. Jiang, H. Zhang, Y. Ren, Z. Han, K.-C. Chen, and L. Hanzo, “Machine learning paradigms for next-generation wireless networks", IEEE Wireless Commun., vol. 24, no. 2, pp. 98-105, Apr. 2017.

[19] R. Li et al., "Intelligent 5G: When cellular networks meet artificial intelligence", IEEE Wireless Commun., vol.24, no.5, pp.175-183, Oct.2017.

[20] Q. Mao, F. Hu, and Q. Hao, “Deep learning for intelligent wireless networks: A comprehensive survey", IEEE Commun. Surveys Tut., vol. 20, no. 4, pp. 2595-2621, 2018.

[21] Ryan C. La Brie and Gerhard H. Steinke, “Towards a Framework for Ethical Audits of AI Algorithms", Twentyfifth Americas Conference on Information Systems, 2019. [Online]. Available: https://aisel.aisnet 